\title{
Effect of chorioamnionitis on brain development and injury in premature newborns
}

\begin{tabular}{|c|c|}
\hline Journal: & Annals of Neurology \\
\hline Manuscript ID: & ANA-08-1322.R2 \\
\hline Wiley - Manuscript type: & Research Article \\
\hline $\begin{array}{r}\text { Date Submitted by the } \\
\text { Author: }\end{array}$ & 19-Mar-2009 \\
\hline Complete List of Authors: & $\begin{array}{l}\text { Chau, Vann; University of British Columbia, Pediatrics } \\
\text { Poskitt, Kenneth; University of British Columbia, Radiology } \\
\text { McFadden, Deborah; University of British Columbia, Pathology } \\
\text { Bowen-Roberts, Tim; University of British Columbia, Pediatrics } \\
\text { Synnes, Anne; University of British Columbia, Pediatrics } \\
\text { Brant, Rollin; University of British Columbia, Statistics } \\
\text { Sargent, Michael; University of British Columbia, Radiology } \\
\text { Soulikias, Wendy; University of British Columbia, Pediatrics } \\
\text { Miller, Steven; University of British Columbia, Department of } \\
\text { Pediatrics }\end{array}$ \\
\hline Domain: & Child Neurology \\
\hline Keywords: & $\begin{array}{l}\text { White matter injury, Magnetic resonance spectroscopy, Diffusion } \\
\text { tensor imaging }\end{array}$ \\
\hline
\end{tabular}

\section{(5) ScholarONE \\ Manuscript Central}


Title: Effect of choriamnionitis on brain development and injury in premature newborns

Vann Chau MD ${ }^{1}$, Kenneth J. Poskitt MDCMㄹ, Deborah E. McFadden MD ${ }^{3}$, Tim BowenRoberts BA ${ }^{1}$, Anne Synnes MDCM${ }^{1}$, Rollin Brant $\mathrm{PhD}^{4}$, Michael A. Sargent MD², Wendy Soulikias RN ${ }^{1}$, Steven P. Miller MAS MDCM ${ }^{1}$

From the Departments of ${ }^{1}$ Pediatrics, ${ }^{2}$ Radiology, ${ }^{3}$ Pathology, and ${ }^{4}$ Statistics, University of British Columbia, Vancouver, Canada

Running Head: Chorioamnionitis and white matter injury

Number of characters in the title: 80 characters

Number of characters in the running head: 40 characters

Number of words in the abstract: 250 words

Number of words in the body of the manuscript: 3824 words

Number of figures: 2 figures ( 1 in grayscale and 1 in color)

Number of tables: 3 tables 


\section{Corresponding Author:}

Steven P. Miller, MAS MDCM FRCPC

British Columbia Children's Hospital

Department of Pediatrics/Division of Neurology - University of British Columbia

K3-180, 4480 Oak Street

Vancouver, British Columbia, V6H 3V4

CANADA

Telephone: 604-875-2345 ext. 5904/ Facsimile: 604-875-2285

E-mail address: smiller6@cw.bc.ca

\section{Keywords (MeSH):}

Placental infection

White matter injury

Magnetic resonance imaging

Diffusion tensor imaging

Magnetic resonance spectroscopic imaging 


\section{ABSTRACT}

\section{Objective}

The association of chorioamnionitis and non-cystic white matter injury, a common brain injury in premature newborns, remains controversial. Our objectives were to determine the association of chorioamnionitis and postnatal risk factors with white matter injury, and the effects of chorioamnionitis on early brain development, using advanced MR imaging.

\section{Methods}

Ninety-two preterm newborns (24-32 weeks gestation) were studied at a median age of 31.9 weeks and again at 40.3 weeks gestation. Histopathological chorioamnionitis and white matter injury were scored using validated systems. Measures of brain metabolism ( $\mathrm{N}$-acetylaspartate/choline and lactate/choline) on magnetic resonance spectroscopy, and microstructure (average diffusivity and fractional anisotropy) on diffusion tensor imaging were calculated from predefined brain regions.

\section{Results}

Thirty-one newborns (34\%) were exposed to histopathological chorioamnionitis, and $26(28 \%)$ had white matter injury. Histopathological chorioamnionitis was not associated with an increased risk of white matter injury (relative risk: $1.2 ; \mathrm{P}=0.6$ ). Newborns with postnatal infections and hypotension requiring therapy were at higher risk of white matter injury $(\mathrm{P}<0.03)$. Adjusting for age at scan and regions of interest, histopathological chorioamnionitis did not significantly affect brain metabolic and microstructural development $(\mathrm{P}>0.1)$. In contrast, white matter injury was associated with 
Chau $/ 4$

lower $\mathrm{N}$-acetylaspartate/choline $(-8.9 \% ; \mathrm{P}=0.009)$ and lower white matter fractional anisotropy $(-11.9 \% ; \mathrm{P}=0.01)$.

\section{Interpretation}

Histopathological chorioamnionitis does not appear to be associated with an increased risk of white matter injury on magnetic resonance imaging or with abnormalities of brain development. In contrast, postnatal infections and hypotension are associated with an increased risk of white matter injury in the premature newborn. 


\section{INTRODUCTION}

Cystic periventricular leukomalacia (PVL) is an important pattern of brain injury in the premature newborn. A meta-analysis found that both clinical and histopathological chorioamnionitis were significantly associated with cystic PVL. ${ }^{1}$ However, the findings were inconsistent among the studies summarized ${ }^{1}$, and the most recent prospective cohort study failed to show an association between histopathological chorioamnionitis and PVL, or brain volumes at term in newborns delivered prematurely. ${ }^{2}$

Over the last decade, the incidence of cystic PVL in premature newborns has decreased dramatically. In contrast, with the increasing use of magnetic resonance (MR) imaging, focal or multifocal non-cystic white matter injury (WMI) is increasingly recognized as the most prevalent pattern of brain injury in premature newborns. ${ }^{3}$ The severity of WMI, best visualized with MRI, is associated with adverse neurodevelopmental outcome at 12-18 months of age. ${ }^{4,5}$ The MR signal changes that characterize this form of WMI (i.e. multifocal WMI) are most easily recognized in the first weeks of life, becoming harder to detect near term-equivalent age. Unfortunately, the risk factors of this spectrum of WMI are poorly understood. Experimental evidence suggests that specific developmentally regulated cell populations prevalent in the white matter of premature newborns, such as late oligodendrocyte progenitor cells and subplate neurons, are vulnerable to oxidative stress and inflammation. ${ }^{6}$ To date, studies have not addressed the association of histopathological chorioamnionitis and WMI on early-life MRI, the time at which WMI is most readily apparent. In order to develop strategies for preventing WMI, it is critical to determine whether prenatal factors, such as 
chorioamnionitis, or postnatal factors, such as hypotension, play significant roles in the pathogenesis of WMI.

The use of advanced MR techniques such as MR spectroscopic imaging (MRSI) and diffusion tensor imaging (DTI) in the first weeks of life now allows the examination of brain development and injury, ${ }^{7}$ at a time closer to in utero exposures, and prior to the development of later neonatal co-morbidities such as chronic lung disease. MRSI measures the concentration of biochemical compounds such as $\mathrm{N}$-acetylaspartate (NAA), choline, and lactate, reflectors of regional brain metabolism. ${ }^{8}$ DTI characterizes the threedimensional spatial distribution of water diffusion in each voxel of the MR image, providing an indirect measure of microstructural development. ${ }^{9-11}$ With maturation, average diffusivity $\left(\mathrm{D}_{\mathrm{AV}}\right)$ decreases presumably due to developing neuronal and glial cell membranes restricting water diffusion. ${ }^{9-11}$ However, fractional anisotropy (FA), the directionality of diffusion, increases with white matter maturation, reflecting the maturation of the oligodendrocyte lineage and early events of myelination. ${ }^{10,12}$

The objectives of this prospective cohort study of premature newborns uniformly studied with placental pathology and advanced MR techniques were to determine: (i) the association of chorioamnionitis and early postnatal risk factors with WMI, (ii) the effects of chorioamnionitis on early brain development as measured by MRSI and DTI. We hypothesized that both chorioamnionitis and postnatal hypotension are important risk factors for WMI, and that exposure to chorioamnionitis would be associated with abnormalities in early brain metabolic and microstructural development. 


\section{MATERIAL AND METHODS}

\section{Study Population}

This study was approved by the University of British Columbia Clinical Research Ethics Board and informed consent was obtained from the parent or legal caregiver of each newborn. The study population consisted of a prospective cohort of preterm newborns born at Children's \& Women's Health Centre of British Columbia, the provincial tertiary-level neonatal referral center, from April 2006 to June 2008. The newborns were eligible if they were delivered between 24-32 weeks gestation. Exclusion criteria comprised: (1) clinical evidence of a congenital malformation or syndrome, (2) antenatal infections, or (3) ultrasound evidence of a large parenchymal hemorrhagic infarction $(>2 \mathrm{~cm}) .{ }^{13}$ Of the eligible newborns, $96(58 \%)$ consented to participate in this study: enrolled newborns were slightly younger (median: 28 versus 29 weeks; $\mathrm{P}<0.001$ ) and had lower birthweight (median: 1045 versus $1268 \mathrm{~g} ; \mathrm{P}<0.001$ ).

\section{Placental Histopathology}

Every placenta was sent fresh to the Pathology Department for macroscopic and microscopic analysis. In addition to gross examination, a minimum of 4 sections were submitted for histopathological examination: reflected membranes, distal and proximal umbilical cord, fetal and maternal surfaces of placenta, and full thickness section. Any grossly identified pathology was also sampled. In the case of multiple gestations, the routine sections were submitted for each infant, in addition to sections of the separating membranes. All tissue sections were fixed in formalin and processed with tissue sections of 3-5 microns thickness cut and stained with hematoxylin and eosin. An experienced placental pathologist (DM) assessed the degree of placental inflammation and scored for 
stage (extent) and grade (severity) of both the maternal and fetal inflammatory responses, using the scale proposed by Redline et al. ${ }^{14}$ For the maternal and fetal inflammatory responses, the stage ranges from early to advanced (score 1 to 3 ) and the grade from mild/moderate (1) to severe (2), according to specific histopathological features. ${ }^{14}$ Additional findings (e.g. infarcts, vascular malformations) were noted.

\section{Magnetic resonance imaging studies}

All newborns were scanned without pharmacological sedation using an MRcompatible isolette (Lammers Medical Technology) and specialized neonatal head coil (Advanced Imaging Research) as soon as they were clinically stable; this provides the shortest interval between in utero exposure and occurrence of focal non-cystic WMI which is most readily apparent on early scans. ${ }^{4}$ The newborns were scanned again at term-equivalent age. The majority of MR scans were of diagnostic quality: T2-weighted images from one term-equivalent scan were excluded for motion. One newborn had its scan repeated as the first attempt was of inadequate quality.

MRI studies were carried out on a Siemens 1.5 Tesla Avanto using VB 13A software and included the following sequences: $3 \mathrm{D}$ coronal volumetric $\mathrm{T}_{1}$-weighted images (TR 36/ TE 9.2/ FOV $200 \mathrm{~mm} /$ Slice thickness $1 \mathrm{~mm} /$ No gap), and axial fast spin echo $\mathrm{T}_{2}$-weighted images (TR 4610/ TE 107/ FOV 160/ Slice thickness 4 mm/ Gap 0.2 $\mathrm{mm})$.

An experienced neuroradiologist (KP) reviewed the images blinded to the newborn's medical history and placental pathology. WMI was defined as foci of abnormal white matter $\mathrm{T}_{1}$ hyperintensity in the absence of marked $\mathrm{T}_{2}$ hypointensity, or by low-intensity $\mathrm{T}_{1}$ foci (cysts). ${ }^{4}$ The severity of WMI was scored as minimal, moderate, or 
severe, using a system found to be predictive of adverse neurodevelopmental outcome 12-18 months of age. ${ }^{4}$ Intraventricular hemorrhage, ventriculomegaly, and cerebellar hemorrhage were also noted. ${ }^{4,13}$ Twenty random scans were re-scored: intra-observer reliability was comparable to that previously reported for these scores (Kappa $>0.9){ }^{4}$

\section{Proton Magnetic Resonance Spectroscopic Imaging}

Magnetic resonance spectroscopic imaging was acquired using multivoxel chemical shift imaging (CSI) (TR 1500/ TE 144/ Averaging 4). The volume of interest [50x50x 10mm(thick)] was placed at 2 levels of the brain: high centrum semi-ovale just above the body of the lateral ventricles (to exclude cerebrospinal fluid) and basal ganglia at the level of the foramen of Monro (Figure 1). All spectra were analyzed off-line by a single observer with voxels $(6 \times 6 \times 10 \mathrm{~mm})$ centered bilaterally on eight predefined anatomical regions (Figure 1). As reported previously, only voxels with adequate signal to noise and fully included in the volume of interest were considered for statistical analyses $(>90 \%) .{ }^{7}$ The mean NAA/choline and lactate/choline ratios were calculated bilaterally for each region of interest. Using Bland Altman analyses, ${ }^{15}$ intra-rater reliability assessed in 15 scans was high: NAA/choline mean difference $<0.001$ (limits of agreement: -0.03 to 0.03 ) and lactate/choline mean difference -0.006 (limits of agreement: -0.06 to 0.05$)$.

\section{Diffusion tensor imaging}

Diffusion tensor imaging was acquired with a multirepetition, single-shot echo planar sequence with 12 gradient directions (TR 4900/ TE 104/ FOV 160 mm/ Slice thickness $3 \mathrm{~mm} /$ No gap), and three averages of two diffusion weightings of 600 and 700 seconds per square millimeter (b value) and an image without diffusion weighting, 
resulting in an in-plane resolution of $1.3 \mathrm{~mm} .{ }^{16}$ The diffusion tensor describes an ellipsoid in space, with size, shape, and orientation given by the "maximum", "intermediate" and "minimum" eigenvalues and their corresponding eigenvectors. The average diffusivity $\left(\mathrm{D}_{\mathrm{AV}}\right)$ reflects the mean of these eigenvalues, expressed as $10^{-3}$ $\mathrm{mm}^{2} / \mathrm{sec}$, whereas the fractional anisotropy (FA) reflects their variance. Parametric maps for $\mathrm{D}_{\mathrm{AV}}$ and FA were generated with values of these DTI parameters calculated by a single observer bilaterally from each of the seven white matter regions as reported previously (Figure 2). ${ }^{16}$ Using Bland Altman analyses, ${ }^{15}$ intra-rater reliability assessed in 15 scans was high: $\mathrm{D}_{\mathrm{AV}}$ mean difference 0.003 (limits of agreement: -0.05 to 0.05 ) and FA mean difference -0.001 (limits of agreement: -0.02 to 0.02 ).

\section{Clinical data collection}

Demographic data and clinical variables were collected systematically. ${ }^{4,17}$ Prolonged premature rupture of membrane (ROM) was defined as ROM $>18$ hours. Clinical chorioamnionitis was defined as a combination of two of: (1) uterine tenderness, (2) maternal and fetal tachycardia, (3) foul-smelling amniotic fluid, (4) maternal fever and leucocytosis in the absence of identified infectious source. Definite episodes of necrotizing enterocolitis (NEC) prior to the first scan were recorded using Bell's criteria. ${ }^{18}$ Postnatal infections prior to the first scan were considered present when cultures were positive in the blood, urine, or cerebrospinal fluid, or if $\geq 4$ white blood cells were found in the tracheal aspirates associated with clinical pneumonia. In the absence of a universally accepted definition, ${ }^{19}$ newborns were considered to have hypotension prior to the first scan if they were treated with saline boluses or vasopressors for low blood pressure. Clinical cranial ultrasound was interpreted using standard criteria 
for cystic PVL. ${ }^{20}$ The clinical condition of the newborns at term equivalent age was described using a neuromotor score as well as respiratory support and feeding route. The neuromotor score, previously found to predict adverse neurodevelopmental outcomes when used at this age, summarizes tone, power, and cranial nerve function, ranging from normal (0) to quadriparesis $(5){ }^{4}$

\section{Data Analysis}

Statistical analysis was performed using Stata 9.2 software (Stata Corporation, College Station, Texas) and $\mathrm{R}^{21}$ Clinical characteristics of the newborns were compared using Fisher's exact test and Mann-Whitney U test for categorical and continuous data respectively. Relative risks with $95 \%$ confidence intervals were calculated to measure the univariate association between histopathological chorioamnionitis and WMI. Logistic regression for repeated measures (generalized estimating equation) was used to account for twin pairing and evaluate the association of histopathological chorioamnionitis and clinical variables with WMI. A linear mixed-effects model, accounting for twin gestation, multiple regions of interest in each infant, and adjusting for postmenstrual age at MRI scan, was used to compare mean values of ratios of NAA and lactate to choline, and $\mathrm{D}_{\mathrm{AV}}$ and FA. The correlation between regions within newborns was described using an unstructured covariance matrix. A random effect for twin pairs was assigned to account for intra-class correlation. A log-transformed outcome variable was used in all regressions to determine the percent differences of the MR measures in newborns with and without chorioamnionitis or WMI. 


\section{RESULTS}

\section{Clinical characteristics of the newborns}

Of the 96 preterm newborns studied, 92 had placental pathology and are described here. Forty-four were born from twin gestations. Newborns were delivered at a median GA of 27.8 weeks (interquartile range: $26.3-29.7$ weeks) and scanned at a median postmenstrual age of 31.9 weeks (IQR: 30.1-33.4 weeks). Seventy-seven newborns were scanned again at "term-equivalent" age [median of 40.3 weeks (IQR: 38.7-42.6 weeks)].

\section{Chorioamnionitis}

Chorioamnionitis was diagnosed histopathologically in 31 (33.7\%) newborns: 13

mild and 18 moderate/severe. The grade and stage of the maternal and fetal inflammatory responses were closely related. When discordant $(\mathrm{N}=12)$, the maternal inflammatory scores were always higher. Funisitis was seen in 16 newborns (17\%). All 6 newborns with clinical chorioamnionitis had histopathological chorioamnionitis: 3 mild and 3 moderate/severe.

Compared to newborns without histopathological chorioamnionitis, those with chorioamnionitis were more likely to be female, have received antenatal antibiotics, and less likely to have pregnancy-induced hypertension (Table 1). While newborns with chorioamnionitis were scanned at a slightly earlier postmenstrual age, the number of days between birth and MRI was not significantly different $(\mathrm{P}=0.1)$.

\section{White matter injury and chorioamnionitis}

WMI was found in $26(28.3 \%)$ newborns: 7 mild, 12 moderate, and 7 severe. Cystic PVL was seen on clinical head ultrasound in 3 newborns. 
Exposure to chorioamnionitis was not associated with an increased risk of WMI [Relative Risk: 1.2 (95\% Confidence Interval: 0.6 to 2.4$)$; $\mathrm{P}=0.6$ ], with an absolute risk difference of $6 \%$. When comparing newborns with moderate to severe WMI to the others in the cohort, histopathological chorioamnionitis was not a significant risk factor [RR: 1.1 (95\% CI: 0.5 to 2.6 ); $\mathrm{P}=0.8$ ] and the absolute risk difference dropped to $3 \%$. Of the three children with cystic PVL, only one had histopathological chorioamnionitis (severe).

The stage and grade of both the maternal and fetal inflammatory responses were similar in the newborns with and without WMI of any severity $(\mathrm{P}>0.1)$ and in newborns with and without moderate to severe WMI $(\mathrm{P}>0.5)$. Funisitis was not a significant risk factor for WMI of any severity or of moderate/severe WMI $(\mathrm{P}>0.1)$. Of the 6 newborns with clinical chorioamnionitis, only one had WMI.

\section{White matter injury on the first MRI and early postnatal risk factors}

Compared to newborns without WMI, those with WMI were less likely to have pregnancy-induced hypertension and C-section delivery, and more likely to have postnatal hypotension requiring intervention (Table 2). Among the 19 newborns exposed to pregnancy-induced hypertension, only one had WMI; 11 of 19 were treated with magnesium sulfate before delivery $(\mathrm{P}<0.001)$. Newborns with and without WMI did not differ significantly with respect to hyaline membrane disease $(88 \% / 79 \%)$, patent ductus arteriosus (58\%/41\%), and definite necrotizing enterocolitis $(11 \% / 3 \%)$ prior to the first scan (all $\mathrm{P}>0.1$ ). Culture-positive postnatal infections prior to the first MRI were more common in newborns with WMI (Table 2). Most of these infections were from staphylococcus species (21); only 3 newborns were infected with Candida, 3 with Escherichia coli, or 2 with others organisms. The occurrence of chronic lung disease was 
not associated with WMI on the first scan $(44 \% / 41 \%$; $\mathrm{P}=0.8)$. Two newborns with WMI (8\%) were conceived by in vitro fertilization, compared to $10(15 \%)$ without WMI $(\mathrm{P}=0.5)$. Of the 22 twin pairs in this cohort, $12(27 \%)$ newborns had WMI with only one twin pair in which both newborns were affected.

In a multivariable model accounting for twin pairs and adjusting for GA at birth, postnatal hypotension requiring intervention was significantly associated with an increased odds of WMI [Odds Ratio: 4.7 (95\% CI: 1.6 to 13.6); $\mathrm{P}=0.004$ ] while histopathological chorioamnionitis was not [OR: 1.6 (95\% CI: 0.4 to 4.0$) ; \mathrm{P}=0.3$ ]. The effects of hypotension or chorioamnionitis were not meaningfully affected by adding either C-section delivery or pregnancy-induced hypertension to the model. Again accounting for twin pairs and adjusting for GA at birth, postnatal infection prior to the first scan was associated with an increased odds of WMI [OR: 4.8 (95\% CI: 1.6 to 14.1); $\mathrm{P}=0.005$ ]. The effect of postnatal infection is attenuated [OR: 3.9 (95\% CI: 1.2 to 12.1$)$; $\mathrm{P}=0.02$ ] when hypotension is added to this model [OR: 4.0 (95\%CI: 1.4 to 11.5$)$; $\mathrm{P}=0.01]$.

\section{Early brain development: chorioamnionitis and white matter injury}

Accounting for twin pairs and adjusting for age at scan and regions of interest, histopathological chorioamnionitis was not significantly associated with NAA/choline or lactate/choline (Table 3a). When restricting the analysis to only the three white matter MRSI regions of interest, the effect of histopathological chorioamnionitis on the metabolite ratios was similar $(\mathrm{P}>0.2)$. In a similar model, histopathological chorioamnionitis was not significantly associated with white matter $F A$ and $D_{A V}$ values. The effect of chorioamnionitis on the MRSI and DTI measures did not differ significantly 
across the regions of interest; no significant interaction is observed between chorioamnionitis and regions of interest for these measures (F-test $\mathrm{P}=0.08-0.7$ for interaction terms).

Accounting for twin pairs and adjusting for age at scan, regions of interest, and histopathological chorioamnionitis, WMI was associated with lower NAA/choline but not lactate/choline (Table 3b). In a similar model, WMI was significantly associated with lower FA but not $\mathrm{D}_{\mathrm{AV}}$ (Table $\left.3 \mathrm{~b}\right)$.

\section{Term-equivalent brain development and injury: effect of chorioamnionitis}

Histopathological chorioamnionitis was not significantly associated with white matter injury on the term-equivalent $\operatorname{scan}(\mathrm{RR}=0.7 ; 95 \% \mathrm{CI}=0.2$ to $1.9 ; \mathrm{P}=0.6)$. Accounting for twin pairs and adjusting for age at scan and regions of interest, histopathological chorioamnionitis was not significantly associated with NAA/choline or lactate/choline ratios (both $\mathrm{P}=0.6)$, or with white matter $\mathrm{FA}(\mathrm{P}=0.4)$ and $\mathrm{D}_{\mathrm{AV}}(\mathrm{P}>0.9)$ on the term-equivalent scan.

\section{Term-equivalent brain injury: effect of postnatal infection}

On the term-equivalent scan, exposure to culture-positive postnatal infections increased the risk of white matter injury, although this effect was not statistically significant $[\mathrm{RR}=2.2 ; 95 \% \mathrm{CI}=0.9$ to $5.2 ; \mathrm{P}=0.08)$. 
Chau $/ 16$

\section{Outcomes}

All newborns in this cohort survived to term-equivalent age with clinical outcomes available in $83(90 \%)$. Neonatal outcomes were similar in newborns with and without histopathological chorioamnionitis (Table 1). In contrast, the newborns with WMI had higher neuromotor scores (i.e. were neurologically more impaired) than the newborns without WMI (Table 2). 


\section{DISCUSSION}

\section{Chorioamnionitis and white matter injury}

In a prospective cohort of premature newborns uniformly assessed with placental pathology and early-life MRI, we find that histopathological chorioamnionitis does not significantly increase the risk of non-cystic white matter injury or abnormalities of brain development in early life or at term-equivalent age. The severity of the maternal and fetal inflammatory responses did not distinguish newborns with and without WMI. To our knowledge, our study is the first to address the association of histopathological chorioamnionitis with brain abnormalities on MRI, including advanced MR techniques, at the time focal non-cystic WMI is most readily apparent. ${ }^{4,17,22}$

Many studies have examined the association of chorioamnionitis with cystic PVL in premature newborns, the most severe pattern of white matter injury. ${ }^{1,2}$ While most individual reports failed to detect an association between chorioamnionitis and cystic PVL, the most recent meta-analysis found both clinical and histopathological chorioamnionitis to be independent risk factors (relative risk of 3.0 and 2.1 respectively). ${ }^{1}$ These authors highlight that the findings from individual studies were often conflicting because of heterogeneous methodologies used to detect chorioamnionitis and brain injury, and suggest the need to adjust for pregnancy-induced hypertension when examining this association. In our cohort, chorioamnionitis was not associated with WMI even after adjusting for pregnancy-induced hypertension. In most studies exposure to chorioamnionitis was diagnosed clinically rather than with placental pathology., ${ }^{23-26}$ Our study highlights the difficulty of clinical diagnosis as only 6 of 31 newborns with histopathological chorioamnionitis met clinical chorioamnionitis criteria. Furthermore, 
most previous studies defined cystic PVL using cranial ultrasonography. ${ }^{12}$ Given the dramatic decline in PVL, and the increasing recognition of WMI on MRI, our failure to detect an association of histopathological chorioamnionitis with the broader spectrum of WMI is relevant to contemporary cohorts. This is important as WMI on MRI is now recognized as an important predictor of early motor and cognitive deficits. ${ }^{4,5}$ Our findings are also consistent with the most recent cohort study where histopathological chorioamnionitis was neither a significant risk for cystic PVL on ultrasound nor smaller brain volumes on MRI at term-equivalent age.

\section{Early brain development: chorioamnionitis and white matter injury}

In our study, histopathological chorioamnionitis was not associated with abnormalities of early brain metabolic or microstructural development. These findings persist to at least term-equivalent age and build on the recent observation that chorioamnionitis does not impact brain volumes. ${ }^{2}$ These data suggest that exposure to chorioamnionitis does not make the premature brain more vulnerable to WMI. In contrast, WMI is associated with early and widespread abnormalities of brain development. This is the first demonstration of the early widespread abnormalities associated with focal WMI lesions on MRI. This finding extends previous observations that newborns with WMI are at higher risk for abnormalities of microstructural ${ }^{10,27}$ and metabolic $^{28}$ brain development, and have smaller brain volumes at term-equivalent age. ${ }^{29-}$

${ }^{31}$ These findings stress the need to prevent or treat WMI in its earliest phase.

\section{Early postnatal factors for white matter injury}

In our cohort, and consistent with earlier reports, hypotension requiring intervention and neonatal infections increased the risk of WMI. ${ }^{32-35}$ The increased risk of 
early WMI with postnatal infection is consistent with the observation that recurrent postnatal sepsis is a risk for progressive WMI. ${ }^{17}$ The increased risk of early WMI with postnatal infection is attenuated by adjusting for hypotension, suggesting that hypotension may be a mechanism by which neonatal infection impacts the brain. It is important to note that our definition of hypotension cannot distinguish the risk of hypotension per se from that of its therapy with saline boluses or vasopressors. ${ }^{36,37}$ Recently, chorioamnionitis has been shown to increase the risk of hypotension in very low birthweight infants on the first postnatal day of life. ${ }^{38}$ Systemic inflammation impairs cerebrovascular autoregulation ${ }^{39}$ and potentiates hypoxic-ischemic insults. ${ }^{40,41}$ While, $^{4}$ preterm newborns with WMI have markers of intrauterine T-cell activation and increased pro-inflammatory cytokines ${ }^{42,43}$, early serum measures of inflammation were not associated with adverse neurodevelopmental outcome in this population. ${ }^{44}$ Given the limitations of current technologies to measure the brain inflammatory response in vivo, cytokine measures were not obtained in our cohort. Our results suggest that chorioamnionitis alone is not associated with WMI. Future studies are warranted to clarify how systemic inflammation, in utero and postnatally, may cause or interact with hypoxia-ischemia in the genesis of WMI.

\section{Limitations}

While the sample size of our cohort may preclude the detection of small differences in the risk of WMI between the newborns with and without chorioamnionitis, we found no difference in MR measures of early brain development. Although the rate of WMI in our study is similar to that reported by others, ${ }^{4,45,46}$ the early MR scans in this cohort may have been obtained prior to the full extent of brain abnormalities related to 
chorioamnionitis being evident. However, no significant association of chorioamnionitis on our measures of brain injury and development were seen at term-equivalent age. Technical limitations of MRSI and DTI may have also precluded detecting a small effect of chorioamnionitis on these brain development measures. The slice thickness of the MRSI may have resulted in some partial volume averaging of unintended structures in the regions of interest. However, these regions' size compare favorably with other neonatal MRSI studies ${ }^{47}$ and are considerably smaller than prior single-voxel MRS studies. ${ }^{8,28}$ A region-of-interest-based approach was used for DTI given the profound changes in brain size and shape over the time period studied, and to our knowledge, tractbased spatial statistic approaches have not yet been validated across this age range. The DTI reliability measures also compare favorably with previous tract-based DTI measures. ${ }^{48}$ The effect of WMI on NAA/choline and FA also suggests any unmeasured effect of chorioamnionitis related to these technical issues would be of lesser magnitude. The association between histopathological chorioamnionitis and neurodevelopmental outcome will be examined as this cohort is followed through childhood. 


\section{Conclusions}

Our data suggest that neonatal infection and hypotension requiring intervention are more significant risk factors for white matter injury than in utero exposure to chorioamnionitis. Importantly, the risk of white matter injury is not directly related to the severity of the fetal and maternal inflammatory responses. Furthermore, while chorioamnionitis is not associated with early abnormalities in brain development, white matter injury is. Clinical trials are needed to determine whether the prevention or treatment of postnatal hypotension reduces the burden of white matter injury in this vulnerable population. 
Chau $/ 22$

Acknowledgment: This work is supported by a Canadian Institutes for Health Research (CIHR) operating grant (CHI 151135). Dr. Chau is supported by the Bourse McLaughlin de l'Université Laval and the Fondation pour la recherche sur les maladies infantiles. Dr. Miller is a Canadian Institutes for Health Research Clinician Scientist and Michael Smith Foundation for Health Research Scholar. We thank Dr. Donna Ferriero at UCSF for her critical review of this manuscript. We also thank the children and their parents who generously participated in this study. The authors have no potential financial conflicts of interest to disclose. 


\section{REFERENCES}

1. Wu YW, Colford JM, Jr. Chorioamnionitis as a risk factor for cerebral palsy: A meta-analysis. Jama 2000;284:1417-1424.

2. Reiman M, Kujari H, Maunu J et al. Does placental inflammation relate to brain lesions and volume in preterm infants? J Pediatr 2008;152:642-647.

3. Hamrick SE, Miller SP, Leonard C et al. Trends in severe brain injury and neurodevelopmental outcome in premature newborn infants: the role of cystic periventricular leukomalacia. J Pediatr 2004;145:593-599.

4. Miller SP, Ferriero DM, Leonard C et al. Early brain injury in premature newborns detected with magnetic resonance imaging is associated with adverse early neurodevelopmental outcome. J Pediatr 2005;147:609-616.

5. Woodward LJ, Anderson PJ, Austin NC et al. Neonatal MRI to predict neurodevelopmental outcomes in preterm infants. N Engl J Med 2006;355:685694.

6. Back SA. Perinatal white matter injury: the changing spectrum of pathology and emerging insights into pathogenetic mechanisms. Ment Retard Dev Disabil Res Rev 2006;12:129-140.

7. Miller SP, McQuillen PS, Hamrick S et al. Abnormal brain development in newborns with congenital heart disease. N Engl J Med 2007;357:1928-1938.

8. Kreis R, Hofmann L, Kuhlmann B et al. Brain metabolite composition during early human brain development as measured by quantitative in vivo $1 \mathrm{H}$ magnetic resonance spectroscopy. Magn Reson Med 2002;48:949-958. 
9. Mukherjee P, Miller JH, Shimony JS et al. Diffusion-tensor MR imaging of gray and white matter development during normal human brain maturation. AJNR Am J Neuroradiol 2002;23:1445-1456.

10. Miller SP, Vigneron DB, Henry RG et al. Serial quantitative diffusion tensor MRI of the premature brain: development in newborns with and without injury. J Magn Reson Imaging 2002;16:621-632.

11. Beaulieu C. The basis of anisotropic water diffusion in the nervous system - a technical review. NMR Biomed 2002;15:435-455.

12. Drobyshevsky A, Song SK, Gamkrelidze G et al. Developmental changes in diffusion anisotropy coincide with immature oligodendrocyte progression and maturation of compound action potential. J Neurosci 2005;25:5988-5997.

13. Papile LA, Burstein J, Burstein R, Koffler H. Incidence and evolution of subependymal and intraventricular hemorrhage: a study of infants with birth weights less than 1,500 gm. J Pediatr 1978;92:529-534.

14. Redline RW, Faye-Petersen O, Heller D et al. Amniotic infection syndrome: nosology and reproducibility of placental reaction patterns. Pediatr Dev Pathol $2003 ; 6: 435-448$.

15. Bland JM, Altman DG. Statistical methods for assessing agreement between two methods of clinical measurement. Lancet 1986;1:307-310.

16. Partridge SC, Mukherjee P, Henry RG et al. Diffusion tensor imaging: serial quantitation of white matter tract maturity in premature newborns. Neuroimage 2004;22:1302-1314. 
17. Glass HC, Bonifacio SL, Chau V et al. Recurrent postnatal infections are associated with progressive white matter injury in premature infants. Pediatrics 2008;122:299-305.

18. Bell MJ, Ternberg JL, Feigin RD et al. Neonatal necrotizing enterocolitis. Therapeutic decisions based upon clinical staging. Ann Surg 1978;187:1-7.

19. Dempsey EM, Barrington KJ. Treating hypotension in the preterm infant: when and with what: a critical and systematic review. J Perinatol 2007;27:469-478.

20. de Vries LS, Eken P, Dubowitz LM. The spectrum of leukomalacia using cranial ultrasound. Behav Brain Res 1992;49:1-6.

21. Team RDC. R: A language and environment for statistical computing. 2.5.0 ed. Vienna: R Foundation for Statistical Computing, 2007

22. Maalouf EF, Duggan PJ, Rutherford MA et al. Magnetic resonance imaging of the brain in a cohort of extremely preterm infants. J Pediatr 1999;135:351-357.

23. Leviton A, Paneth N, Reuss ML et al. Maternal infection, fetal inflammatory response, and brain damage in very low birth weight infants. Developmental Epidemiology Network Investigators. Pediatr Res 1999;46:566-575.

24. Kaukola T, Herva R, Perhomaa M et al. Population cohort associating chorioamnionitis, cord inflammatory cytokines and neurologic outcome in very preterm, extremely low birth weight infants. Pediatr Res 2006;59:478-483.

25. Rocha G, Proenca E, Quintas C et al. Chorioamnionitis and brain damage in the preterm newborn. J Matern Fetal Neonatal Med 2007;20:745-749. 
26. Murata Y, Itakura A, Matsuzawa K et al. Possible antenatal and perinatal related factors in development of cystic periventricular leukomalacia. Brain Dev 2005;27:17-21.

27. Huppi PS, Murphy B, Maier SE et al. Microstructural brain development after perinatal cerebral white matter injury assessed by diffusion tensor magnetic resonance imaging. Pediatrics 2001;107:455-460.

28. Robertson NJ, Kuint J, Counsell TJ et al. Characterization of cerebral white matter damage in preterm infants using $1 \mathrm{H}$ and $31 \mathrm{P}$ magnetic resonance spectroscopy. J Cereb Blood Flow Metab 2000;20:1446-1456.

29. Inder TE, Warfield SK, Wang $\mathrm{H}$ et al. Abnormal cerebral structure is present at term in premature infants. Pediatrics 2005;115:286-294.

30. Thompson DK, Warfield SK, Carlin JB et al. Perinatal risk factors altering regional brain structure in the preterm infant. Brain 2007;130:667-677.

31. Srinivasan L, Dutta R, Counsell SJ et al. Quantification of deep gray matter in preterm infants at term-equivalent age using manual volumetry of 3-tesla magnetic resonance images. Pediatrics 2007;119:759-765.

32. Weindling AM, Wilkinson AR, Cook J et al. Perinatal events which precede periventricular haemorrhage and leukomalacia in the newborn. Br J Obstet Gynaecol 1985;92:1218-1223.

33. Miall-Allen VM, de Vries LS, Whitelaw AG. Mean arterial blood pressure and neonatal cerebral lesions. Arch Dis Child 1987;62:1068-1069.

34. Watkins AM, West CR, Cooke RW. Blood pressure and cerebral haemorrhage and ischaemia in very low birthweight infants. Early Hum Dev 1989;19:103-110. 
35. Low JA, Froese AB, Galbraith RS et al. The association between preterm newborn hypotension and hypoxemia and outcome during the first year. Acta Paediatr 1993;82:433-437.

36. Limperopoulos C, Bassan H, Kalish LA et al. Current definitions of hypotension do not predict abnormal cranial ultrasound findings in preterm infants. Pediatrics 2007;120:966-977.

37. Barrington KJ. Hypotension and shock in the preterm infant. Semin Fetal Neonatal Med 2008;13:16-23.

38. Lee SY, Ng DK, Fung GP et al. Chorioamnionitis with or without funisitis increases the risk of hypotension in very low birthweight infants on the first postnatal day but not later. Arch Dis Child Fetal Neonatal Ed 2006;91:F346-348.

39. Yanowitz TD, Potter DM, Bowen A et al. Variability in cerebral oxygen delivery is reduced in premature neonates exposed to chorioamnionitis. Pediatr Res 2006;59:299-304.

40. Larouche A, Roy M, Kadhim $\mathrm{H}$ et al. Neuronal injuries induced by perinatal hypoxic-ischemic insults are potentiated by prenatal exposure to lipopolysaccharide: animal model for perinatally acquired encephalopathy. Dev Neurosci 2005;27:134-142.

41. Wang $\mathrm{X}$, Hagberg $\mathrm{H}, \mathrm{Nie} \mathrm{C}$ et al. Dual role of intrauterine immune challenge on neonatal and adult brain vulnerability to hypoxia-ischemia. J Neuropathol Exp Neurol 2007;66:552-561. 
42. Duggan PJ, Maalouf EF, Watts TL et al. Intrauterine T-cell activation and increased proinflammatory cytokine concentrations in preterm infants with cerebral lesions. Lancet 2001;358:1699-1700.

43. Ellison VJ, Mocatta TJ, Winterbourn CC et al. The relationship of CSF and plasma cytokine levels to cerebral white matter injury in the premature newborn. Pediatr Res 2005;57:282-286.

44. Nelson KB, Grether JK, Dambrosia JM et al. Neonatal cytokines and cerebral palsy in very preterm infants. Pediatr Res 2003;53:600-607.

45. Cornette LG, Tanner SF, Ramenghi LA et al. Magnetic resonance imaging of the infant brain: anatomical characteristics and clinical significance of punctate lesions. Arch Dis Child Fetal Neonatal Ed 2002;86:F171-177.

46. Dyet LE, Kennea N, Counsell SJ et al. Natural history of brain lesions in extremely preterm infants studied with serial magnetic resonance imaging from birth and neurodevelopmental assessment. Pediatrics 2006;118:536-548.

47. Vigneron DB, Barkovich AJ, Noworolski SM et al. Three-dimensional proton MR spectroscopic imaging of premature and term neonates. AJNR Am J Neuroradiol 2001;22:1424-1433.

48. Partridge SC, Mukherjee P, Berman JI et al. Tractography-based quantitation of diffusion tensor imaging parameters in white matter tracts of preterm newborns. $\mathbf{J}$ Magn Reson Imaging 2005;22:467-474.

49. Pajevic S, Pierpaoli C. Color schemes to represent the orientation of anisotropic tissues from diffusion tensor data: application to white matter fiber tract mapping in the human brain. Magn Reson Med 1999;42:526-540. 
Figure 1 Proton magnetic resonance imaging and regions of interest

Figure 1 shows the magnetic resonance (MR) spectroscopic imaging and the 8 regions of interest that were analyzed in a premature newborn with a normal MR imaging and born at $26^{+6 / 7}$ weeks gestation and scanned at $29^{+3 / 7}$ weeks postmenstrual age, at the level of (A) the high centrum semi-ovale and (B) the basal ganglia. The values of each region were averaged bilaterally: high white matter [(1) anterior, (2) central, and (3) posterior], (4) caudate, (5) lentiform nuclei, (6) thalamus, (7) optic radiations, (8) calcarine region. The spectrum of the right thalamus is shown in $\mathrm{C}$.

Legend: $\mathrm{Cho}=\mathrm{Choline} ; \mathrm{Cr}=\mathrm{Creatine} ; \mathrm{NAA}=\mathrm{N}$-acetylaspartate; Lac $=$ Lactate.

Figure 2 Diffusion tensor imaging and regions of interest

Figure 2 shows the axial diffusion tensor imaging encoded anisotropy color map and the 7 regions of interest that were analyzed in a premature newborn with a normal MR imaging and born at $26^{+6 / 7}$ weeks gestation and scanned at $29^{+3 / 7}$ weeks postmenstrual age, at (A) the high centrum semi-ovale and (B) the basal ganglia. The values of each region were averaged bilaterally: high white matter [(1) anterior, (2) central, (3) posterior], (4) genu of the corpus callosum, (5) posterior limb of the internal capsule, (6) splenium of the corpus callosum, and (7) optic radiations. The color convention used to display the predominant diffusion direction has red representing right-left, green representing anterior-posterior, and blue representing superior-inferior anatomical directions. ${ }^{16,49}$ 
Table 1 Demographic and clinical characteristics of the newborns with and without histopathological chorioamnionitis (univariate analysis)

\begin{tabular}{|c|c|c|c|}
\hline \multirow{2}{*}{$\begin{array}{l}\text { Number (\%) or Median } \\
\text { (interquartile range) }\end{array}$} & \multicolumn{3}{|c|}{ Histopathological chorioamnionitis } \\
\hline & Yes & No & $\mathbf{P}$ \\
\hline Number & $31(34 \%)$ & $61(66 \%)$ & \\
\hline Male sex & $9(29 \%)$ & $35(57 \%)$ & 0.02 \\
\hline GA at birth (weeks) & $27.3(26.3-28.1)$ & $28.6(26.0-30.0)$ & 0.1 \\
\hline PMA at first scan (weeks) & $30.3(29.4-31.4)$ & $32.4(31.3-34.0)$ & $<0.001$ \\
\hline Birth weight (grams) & $1035(810-1235)$ & $1095(835-1285)$ & 0.9 \\
\hline Head circumference $(\mathrm{cm})$ & $25.3(23.5-26.0)$ & $26.1(24.1-28.0)$ & 0.2 \\
\hline PIH & 0 & $19(31 \%)$ & 0.001 \\
\hline Antenatal antibiotics & $27(87 \%)$ & $32(53 \%)$ & 0.001 \\
\hline Antenatal steroids & $23(74 \%)$ & $37(62 \%)$ & 0.3 \\
\hline C-section delivery & $15(48 \%)$ & $38(62 \%)$ & 0.3 \\
\hline SNAP-PE & $24(9-40)$ & $18(9-36)$ & 0.6 \\
\hline Definite NEC & $1(3 \%)$ & $4(7 \%)$ & 0.7 \\
\hline $\begin{array}{l}\text { Culture positive postnatal } \\
\text { infection }\end{array}$ & $8(26 \%)$ & $21(34 \%)$ & 0.5 \\
\hline Neonatal hypotension & $9(29 \%)$ & $20(33 \%)$ & 0.8 \\
\hline \multicolumn{4}{|l|}{ Brain abnormalities } \\
\hline WMI & $10(32 \%)$ & $16(26 \%)$ & 0.6 \\
\hline$I V H$ & $11(35 \%)$ & $29(48 \%)$ & 0.4 \\
\hline Ventriculomegaly & $10(32 \%)$ & $13(21 \%)$ & 0.5 \\
\hline
\end{tabular}




\begin{tabular}{|r|c|c|c|}
\hline Cerebellar hemorrhage & $6(19 \%)$ & $4(7 \%)$ & 0.08 \\
\hline equivalent-age & & & \\
\hline GA at assessment (weeks) & $40.3(38.0-41.3)$ & $40.3(39.1-42.6)$ & 0.5 \\
\hline Neuromotor score & $1(0-2)$ & $1(0-2)$ & 0.5 \\
\hline NGfeeds & 9 & 18 & 0.4 \\
\hline CPAP & 1 & 4 & $>0.9$ \\
\hline
\end{tabular}

$\mathrm{GA}=$ Gestational age

PMA $=$ Postmenstrual age

PIH=Pregnancy-induced hypertension

ROM=Rupture of membranes

SNAP-PE=Score of neonatal acute physiology- perinatal extension

$\mathrm{NEC}=$ Necrotizing enterocolitis

WMI=White matter injury

IVH=Intraventricular hemorrhage

$\mathrm{NG}=$ Nasogastric

$\mathrm{CPAP}=\mathrm{Continuous} \mathrm{positive} \mathrm{airway} \mathrm{pressure}$

*Neonatal outcomes available for $90 \%$ of the cohort 
Table 2 Demographic and clinical characteristics of the newborns with and without white matter injury on the first scan (univariate analysis)

\begin{tabular}{|c|c|c|c|}
\hline \multirow{2}{*}{$\begin{array}{l}\text { Number }(\%) \text { or Median } \\
\text { (interquartile range) }\end{array}$} & \multicolumn{3}{|c|}{ White matter injury } \\
\hline & Yes & No & $\mathbf{P}$ \\
\hline Number & $26(28 \%)$ & $66(72 \%)$ & \\
\hline Male sex & $11(42 \%)$ & $33(50 \%)$ & 0.6 \\
\hline GA at birth (weeks) & $27.9(26.4-29.7)$ & $27.6(26.2-29.7)$ & 0.9 \\
\hline PMA at first scan (weeks) & $31.6(30.1-32.4)$ & $32.0(30.1-33.6)$ & 0.6 \\
\hline Birth weight (grams) & $1150(910-1330)$ & $1045(813-1230)$ & 0.2 \\
\hline Head circumference $(\mathrm{cm})$ & $25.5(25.0-28.0)$ & $25.3(23.5-27.0)$ & 0.2 \\
\hline PIH & $1(4 \%)$ & $18(27 \%)$ & 0.03 \\
\hline Antenatal antibiotics & $16(62 \%)$ & $43(65 \%)$ & $>0.9$ \\
\hline Antenatal steroids & $16(62 \%)$ & $44(67 \%)$ & 0.8 \\
\hline C-section delivery & $10(38 \%)$ & $43(65 \%)$ & 0.03 \\
\hline SNAP-PE & $26(15-57)$ & $18(9-32)$ & 0.1 \\
\hline Definite NEC & $3(11 \%)$ & $2(3 \%)$ & 0.1 \\
\hline $\begin{array}{l}\text { Culture positive postnatal } \\
\text { infection }\end{array}$ & $13(50 \%)$ & $16(24 \%)$ & 0.03 \\
\hline Neonatal hypotension & $14(54 \%)$ & $15(23 \%)$ & 0.006 \\
\hline $\begin{array}{l}\text { Histopathological } \\
\text { chorioamnionitis }\end{array}$ & $10(38 \%)$ & $21(32 \%)$ & 0.6 \\
\hline \multicolumn{4}{|l|}{ Other brain abnormalities } \\
\hline IVH & $14(54 \%)$ & $26(39 \%)$ & 0.2 \\
\hline
\end{tabular}




\begin{tabular}{|r|c|c|c|}
\hline $\begin{array}{r}\text { Ventriculomegaly } \\
\text { Cerebellar hemorrhage }\end{array}$ & $11(42 \%)$ & $12(18 \%)$ & 0.03 \\
\hline $\begin{array}{r}\text { Outcome* at term- } \\
\text { equivalent age }\end{array}$ & $4(15 \%)$ & $6(9 \%)$ & 0.5 \\
\hline GA at assessment & $40.4(38.6-43.0)$ & $40.3(38.7-42.3)$ & $>0.9$ \\
\hline Neuromotor score & $2(2-3)$ & $1(0-1)$ & $<0.001$ \\
\hline NG feeds & 5 & 18 & 0.1 \\
\hline CPAP & 1 & 4 & 0.3 \\
\hline
\end{tabular}

$\mathrm{GA}=$ Gestational age

PMA $=$ Postmenstrual age

$\mathrm{PIH}=$ Pregnancy-induced hypertension

ROM=Rupture of membranes

SNAP-PE=Score of neonatal acute physiology- perinatal extension

$\mathrm{NEC}=$ Necrotizing enterocolitis

$\mathrm{IVH}=$ Intraventricular hemorrhage

$\mathrm{NG}=\mathrm{Nasog}$ astric

$\mathrm{CPAP}=\mathrm{Continuous} \mathrm{positive} \mathrm{airway} \mathrm{pressure}$

*Neonatal outcomes available for $90 \%$ of the cohort 
Chau / 34

Table 3 Comparison of metabolic and microstructural brain development on the early

MR scan

A. Newborns without and with histopathological chorioamnionitis

\begin{tabular}{|c|c|c|c|c|}
\hline Mean & Without & With & Adjusted difference ** & P \\
& histopathological & histopathological & (95\% CI) & \\
\hline MRSI & 0.450 & 0.435 & $-3.8 \%(-10.9$ to +3.9$)$ & 0.3 \\
NAA/choline & 0.158 & 0.148 & $-12.6 \%(-26.2$ to +3.5$)$ & 0.1 \\
Lactate/choline & 1.431 & 1.446 & $+1.1 \%(-5.3$ to +7.9$)$ & 0.7 \\
\hline DTI & 0.270 & 0.284 & $+5.4 \%(-4.9$ to 16.8$)$ & 0.3 \\
FA & & & & 0.3 \\
\hline
\end{tabular}


B. Newborns without and with white matter injury

\begin{tabular}{|l|c|c|c|c|}
\hline Mean & $\begin{array}{c}\text { Without white } \\
\text { matter injury * }\end{array}$ & $\begin{array}{c}\text { With white } \\
\text { matter injury * }\end{array}$ & $\begin{array}{c}\text { Adjusted difference ** } \\
\%(95 \% \mathrm{CI})\end{array}$ & $\mathrm{P}$ \\
\hline MRSI & 0.448 & 0.408 & $-8.9 \%(-14.9$ to -2.6$)$ & 0.009 \\
NAA/choline & 0.159 & 0.150 & $-9.9 \%(-21.9$ to +8.7$)$ & 0.3 \\
Lactate/choline & 1.418 & 1.488 & $+2.8 \%(-1.0$ to +6.8$)$ & 0.1 \\
\hline DTI & 0.282 & 0.251 & $-11.9 \%(-20.0$ to -2.9$)$ & 0.01 \\
\hline FA & & & & \\
\hline
\end{tabular}

* Mean values on the early scan across all brain regions, adjusted for gestational age at scan

**Analyses were adjusted for gestational age at the time of early MRI, and accounted for multiple brain regions in an individual newborn and twin pairs

95\% CI=95\% confidence interval

MRSI=Magnetic resonance spectroscopic imaging

DTI=Diffusion tensor imaging

$\mathrm{NAA}=\mathrm{N}$-acetylaspartate

$D_{A v}=$ Average diffusivity

FA=Fractional anisotropy 


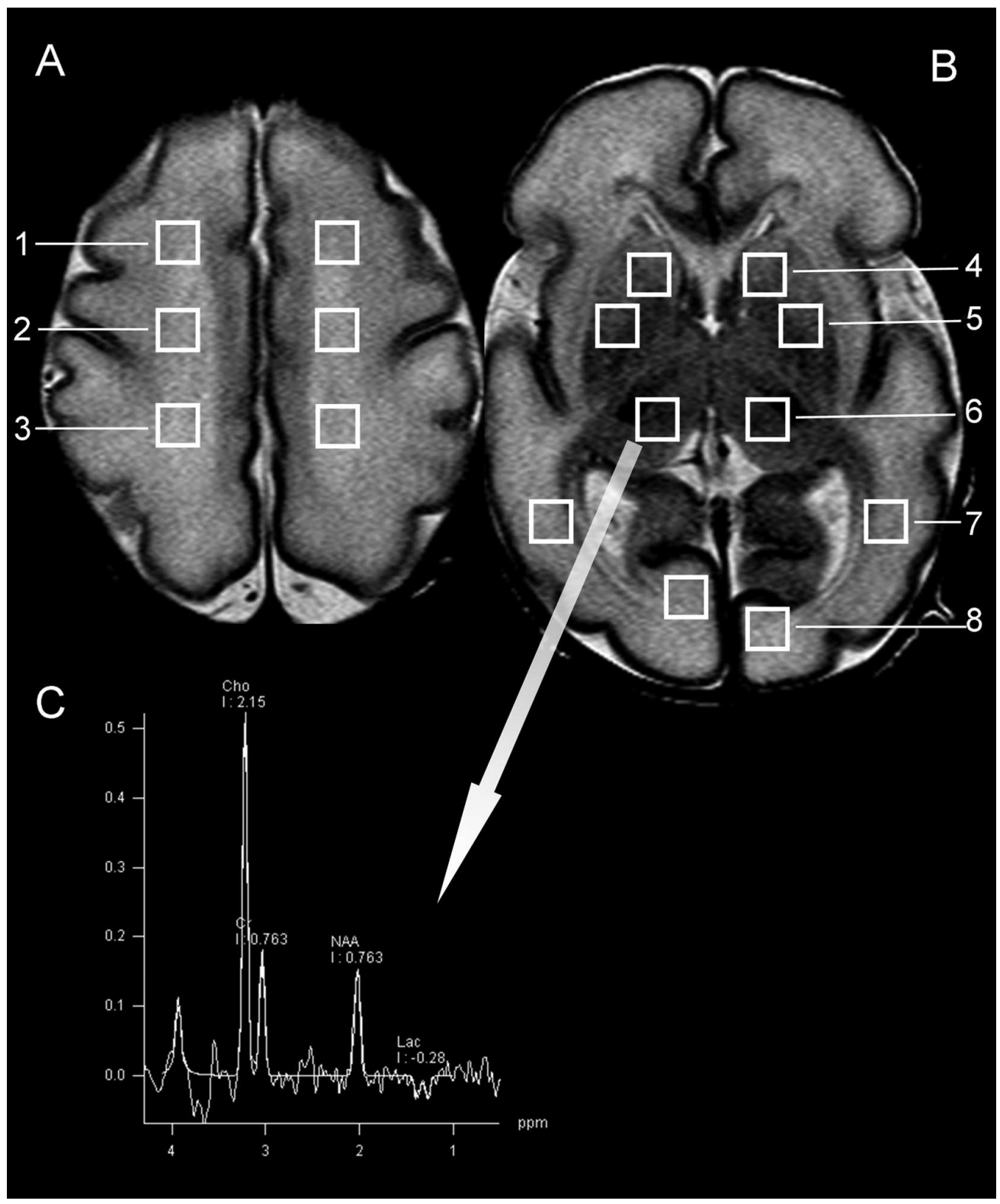

Figure 1 Proton magnetic resonance imaging and regions of interest

Figure 1 shows the magnetic resonance (MR) spectroscopic imaging and the 8 regions of interest that were analyzed in a premature newborn with a normal MR imaging and born at $26+6 / 7$ weeks gestation and scanned at 29+3/7 weeks postmenstrual age, at the level of $(A)$ the high centrum semi-ovale and (B) the basal ganglia. The values of each region were averaged bilaterally: high white matter [(1) anterior, (2) central, and (3) posterior], (4) caudate, (5) lentiform nuclei, (6) thalamus, (7) optic radiations, (8) calcarine region. The spectrum of the right thalamus is shown in C.

Legend: $\mathrm{Cho}=$ Choline; $\mathrm{Cr}=$ Creatine; $\mathrm{NAA}=\mathrm{N}$-acetylaspartate; Lac $=$ Lactate. $70 \times 84 \mathrm{~mm}(600 \times 600 \mathrm{DPI})$ 


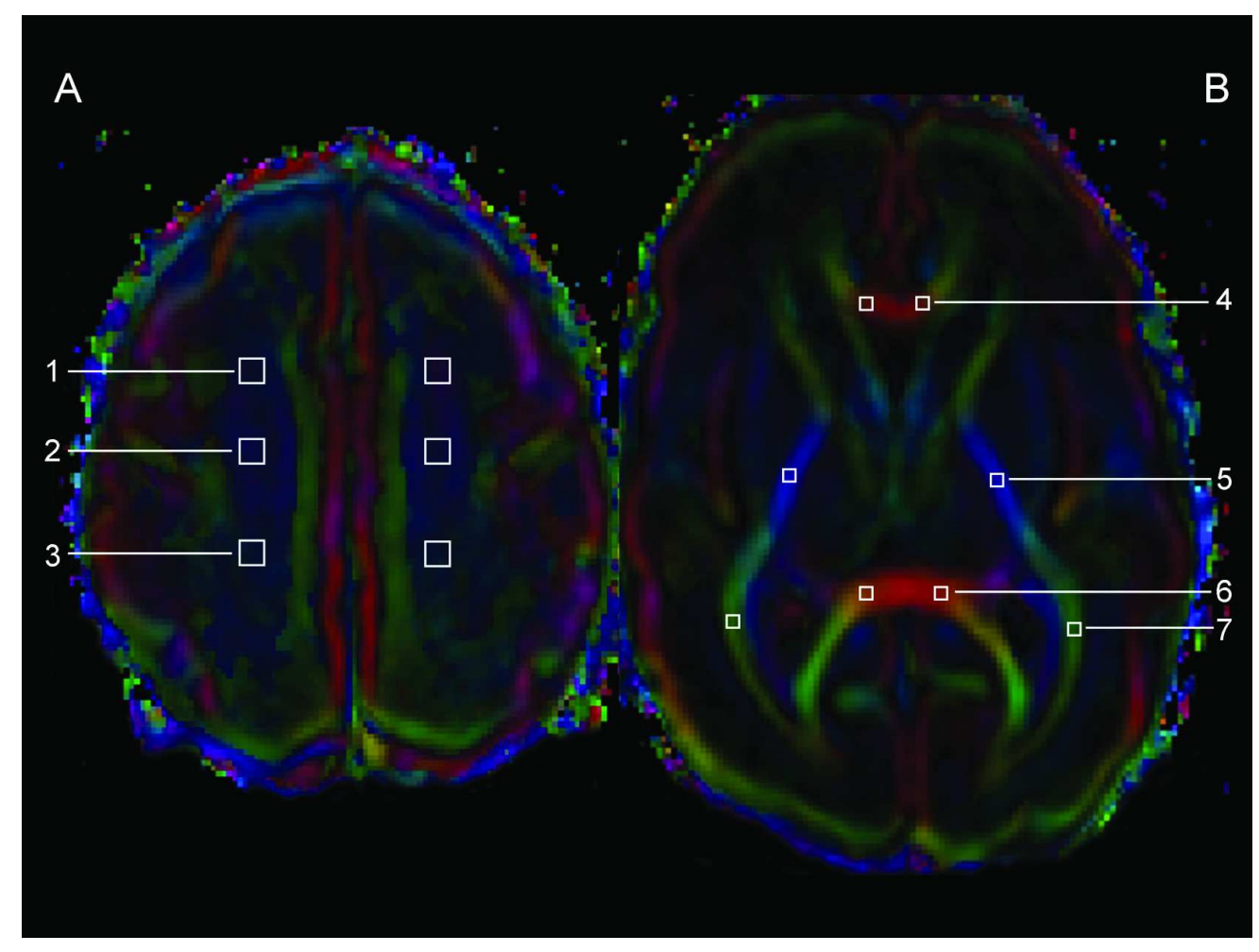

Figure 2 Diffusion tensor imaging and regions of interest

Figure 2 shows the axial diffusion tensor imaging encoded anisotropy color map and the 7 regions of interest that were analyzed in a premature newborn with a normal MR imaging and born at $26+6 / 7$ weeks gestation and scanned at $29+3 / 7$ weeks postmenstrual age, at $(A)$ the high centrum semiovale and $(B)$ the basal ganglia. The values of each region were averaged bilaterally: high white matter [(1) anterior, (2) central, (3) posterior], (4) genu of the corpus callosum, (5) posterior limb of the internal capsule, (6) splenium of the corpus callosum, and (7) optic radiations. The color convention used to display the predominant diffusion direction has red representing right-left, green representing anterior-posterior, and blue representing superior-inferior anatomical directions. $70 \times 52 \mathrm{~mm}(600 \times 600 \mathrm{DPI})$ 